\title{
Microvascular effects of esmolol in postoperative atrial fibrillation: a prospective pilot study in cardiac surgery
}

\author{
W. Fornier ${ }^{\prime, 2}$, M. Jacquet-Lagreze', T.Collenot ', P.Teixeira', P. Portran', R. Schweizer', \\ M. Ovize ${ }^{2,3}$, JL. Fellahil,2 \\ 'Department of Anesthesiology and Intensive care, University Hospital Louis Pradel, Lyon, France; \\ ${ }^{2}$ Inserm U 1060, Faculty of Medicine, Claude Bernard Lyon I University, Lyon, France \\ ${ }^{3}$ Department of Cardiology, University Hospital Louis Pradel, Lyon, France
}

\section{Background and goal of study}

Postoperative atrial fibrillation (POAF) is commonplace after cardiothoracic surgery. Rate control with betablockers is recommended as a first-line therapy in patients macro-

hemodynamically stable. Esmolol is a short-acting $\beta I$-selective betablocker whose microcirculatory effects have not been yet investigated.We tested the hypothesis that esmolol could improve the POAF-related microvascular dysfunction.

\section{Materials and Methods}

After approval of the Ethical Committee, we prospectively included 20 cardiothoracic surgical patients with POAF in the Teaching University Hospital Louis Pradel (Lyon, France). Microcirculation was assessed by near-infrared spectroscopy (NIRS) and a vascular occlusion test (VOT) at successive time points: before esmolol infusion, during incremental doses of esmolol $(25,50,100$, and $200 \mu \mathrm{g} / \mathrm{kg} / \mathrm{min})$ and after return to sinus rhythm. Esmolol dose regimen was increased every 45 minutes until heart rate reached a targeted value between 60 and 90 beats $/ \mathrm{min}$. NIRS parameters included $\mathrm{StO}_{2}, \mathrm{StO}_{2}$ min, $\mathrm{StO}_{2} \max , \Delta \mathrm{StO}_{2}$, and both

desaturation and resaturation speeds. Data before and after

cardioversion were compared with a paired Student $t$ test or a Wilcoxon test. A one-way ANOVA for repeated measurements or a Friedman test were used to compare the effects of incremental doses of esmolol.

\section{$\underline{\text { Results and discussion }}$}

$\mathrm{StO}_{2}$ and resaturation speed were significantly improved between POAF and return to sinus rhythm: $\mathrm{StO}_{2} 64.5 \% \pm 3.9$ vs. $67.0 \% \pm 5.1$, $\mathrm{P}=0.047$; and resaturation speed $0.6 \% / \mathrm{s}(0.45-0.99)$ vs. $0.7 \% / \mathrm{s}(0.50$ -

I.03), $\mathrm{p}=0.02 . \Delta \mathrm{StO}_{2}$ was significantly lower after return to sinus rhythm, $8.0 \pm 4.5$ vs. $6.0 \pm 4.5, p=0.026$.

During esmolol infusion, $\mathrm{StO}_{2}$ and resaturation speeds were not statistically different (Figure I). No significant difference was found at any other time point for $\mathrm{StO}_{2} \mathrm{~min}, \mathrm{StO}_{2} \max , \Delta \mathrm{StO}_{2}$ and desaturation speed. On the other hand, mean arterial pressure showed a trend toward a decrease $(p=0.054)$ whereas pulse pressure had a significant reduction $(p<0.001)$. Heart rate was lowered as expected $(p=$ $0.031)$.
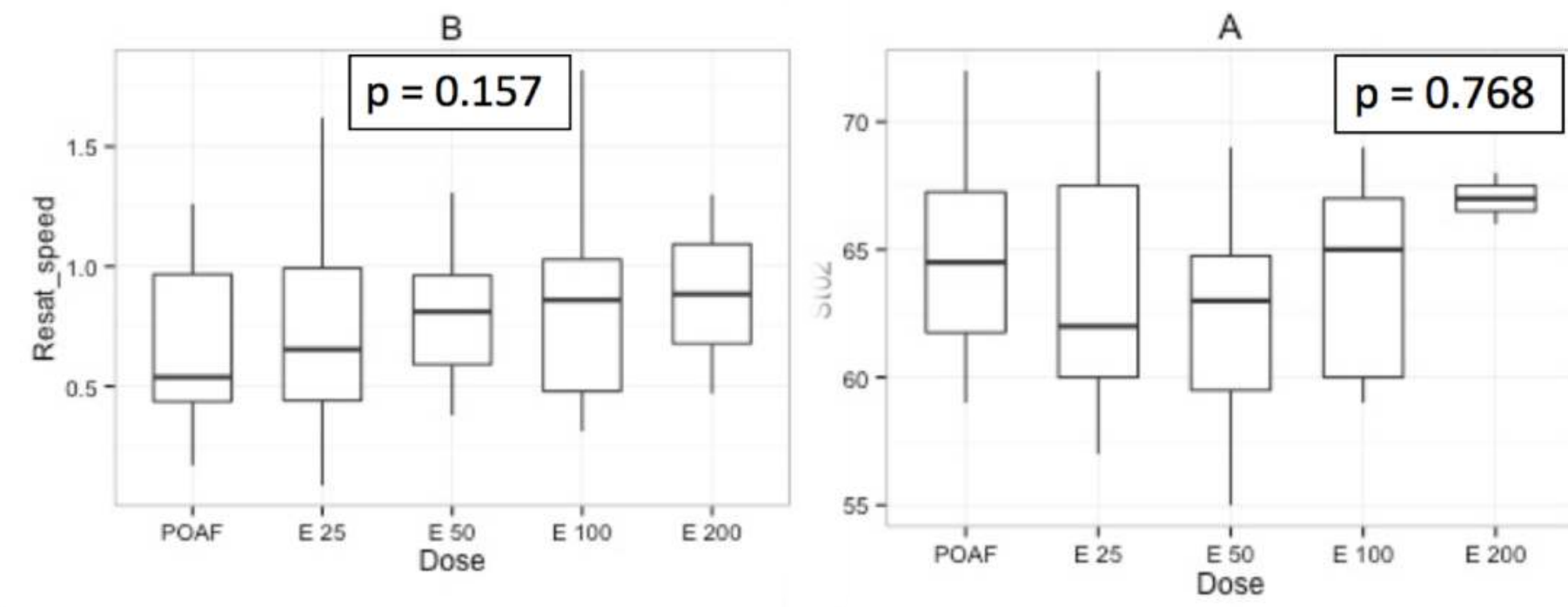

Figure I: Boxplots from microcirculation values during POAF and its treatment with esmolol. Continuous variables were analyzed with a repeated measures analysis of variance (ANOVA test) and a Friedman test, depending on their homogeneity of variances. (A) $\mathrm{StO}_{2}$, (B) Resaturation speed.

Impact of spontaneous cardioversion on macro and microcirculation variables

\begin{tabular}{cccc}
\hline VARIABLES & POAF & SR & p value \\
\hline HR (beats per & $133(116-139)$ & $74(67-84)$ & 0.001 \\
minute) & & & \\
$\mathrm{MAP}(\mathrm{mmHg})$ & $79 \pm 12.3$ & $80 \pm 12.5$ & 0.897 \\
$\mathbf{P P}$ (mmHg) & $51 \pm 14$ & $59 \pm 15$ & $<0.001$ \\
$\mathrm{SpO}_{2}$ (\%) & $95(94-96)$ & $97(96-98)$ & 0.046 \\
$\mathrm{StO}_{2}$ (\%) & $64.5 \pm 3.9$ & $67.0 \pm 5.1$ & 0.047 \\
Resaturation speed & $0.62(0.46-0.99)$ & $0.66(0.50-$ & 0.020 \\
(\%/sec.) & & $1.03)$ & \\
Desaturation speed & $0.08(0.05-0.09)$ & $0.09(0.05-$ & 0.529 \\
(\%/sec.) & & $0.10)$ & \\
$\Delta$ StO $_{2}$ (\%) & $8.0 \pm 4.5$ & $6.0 \pm 4.5$ & 0.026 \\
StO $_{2}$ min (\%) & $40(39-41)$ & $40(40-46)$ & 0.396 \\
StO $_{2}$ max (\%) & $73(71-77)$ & $73(71-76)$ & 0.483 \\
\hline
\end{tabular}

Data are absolute values, means \pm SD or medians ( 25 th and 75 th percentiles) Continuous variables were analyzed with a paired Student $t$ test and a

Wilcoxon test, according to distribution. HR: heart rate, MAP: mean arterial pressure, $\mathrm{PP}:$ pulse pressure, $\mathrm{SpO}_{2}$ : Saturation of peripheral oxygen.

\section{Conclusion}

Stable hemodynamic POAF is associated with significant microvascular dysfunction. Rate control with intravenous esmolol showed a not significant trend toward a dose-dependent improvement in POAFrelated microvascular dysfunction.

\section{Keywords}

